\title{
Growth-uncoupled isoprenoid synthesis in Rhodobacter sphaeroides
}

\author{
Enrico Orsi ${ }^{1,6+}{ }^{0}$, loannis Mougiakos ${ }^{2,7 \dagger}$, Wilbert Post ${ }^{1,2}$, Jules Beekwilder ${ }^{3}$, Marco Dompè ${ }^{4}$, Gerrit Eggink ${ }^{1,5}$, \\ John van der Oost ${ }^{2}$, Servé W. M. Kengen² and Ruud A. Weusthuis ${ }^{1 *}$
}

\begin{abstract}
Background: Microbial cell factories are usually engineered and employed for cultivations that combine product synthesis with growth. Such a strategy inevitably invests part of the substrate pool towards the generation of biomass and cellular maintenance. Hence, engineering strains for the formation of a specific product under non-growth conditions would allow to reach higher product yields. In this respect, isoprenoid biosynthesis represents an extensively studied example of growth-coupled synthesis with rather unexplored potential for growth-independent production. Rhodobacter sphaeroides is a model bacterium for isoprenoid biosynthesis, either via the native 2-methyl-D-erythritol 4-phosphate (MEP) pathway or the heterologous mevalonate (MVA) pathway, and for poly- $\beta$-hydroxybutyrate (PHB) biosynthesis.

Results: This study investigates the use of this bacterium for growth-independent production of isoprenoids, with amorpha-4,11-diene as reporter molecule. For this purpose, we employed the recently developed Cas9-based genome editing tool for $R$. sphaeroides to rapidly construct single and double deletion mutant strains of the MEP and PHB pathways, and we subsequently transformed the strains with the amorphadiene producing plasmid. Furthermore, we employed ${ }^{13} \mathrm{C}-$ metabolic flux ratio analysis to monitor the changes in the isoprenoid metabolic fluxes under different cultivation conditions. We demonstrated that active flux via both isoprenoid pathways while inactivating PHB synthesis maximizes growth-coupled isoprenoid synthesis. On the other hand, the strain that showed the highest growth-independent isoprenoid yield and productivity, combined the plasmid-based heterologous expression of the orthogonal MVA pathway with the inactivation of the native MEP and PHB production pathways.
\end{abstract}

Conclusions: Apart from proposing a microbial cell factory for growth-independent isoprenoid synthesis, this work provides novel insights about the interaction of MEP and MVA pathways under different growth conditions.

Keywords: Rhodobacter sphaeroides, Isoprenoid biosynthesis, PHB, MEP, MVA, Growth-independent production

\section{Background}

Isoprenoids (also known as terpenoids) have great industrial value as ingredients of pharmaceuticals, perfumes, food flavourings and most recently biofuels [1-6]. They are formed by the condensation of the five-carbon monomers isopentenyl pyrophosphate (IPP) and its isomer

\footnotetext{
*Correspondence: ruud.weusthuis@wur.nl

${ }^{\dagger}$ Enrico Orsi and loannis Mougiakos contributed equally to this work

${ }^{1}$ Bioprocess Engineering, Wageningen University, Droevendaalsesteeg 1, 6708 PB Wageningen, The Netherlands

Full list of author information is available at the end of the article
}

dimethylallyl pyrophosphate (DMAPP). The two naturally existing IPP/DMAPP production pathways are the 2-C-methylerythritol 4-phosphate (MEP) pathway and the mevalonate (MVA) pathway [7]. While the former branches in the central metabolism from glyceraldehyde 3-phosphate and pyruvate, the latter uses acetoacetylCoA (AA-CoA) as precursor (Fig. 1). These two pathways are, with few exceptions, phylogenetically distinct: the MEP pathway is present in prokaryotes, while the MVA pathway is found in archaea and eukaryotes [8]. Plants express both metabolic routes, with the MEP 


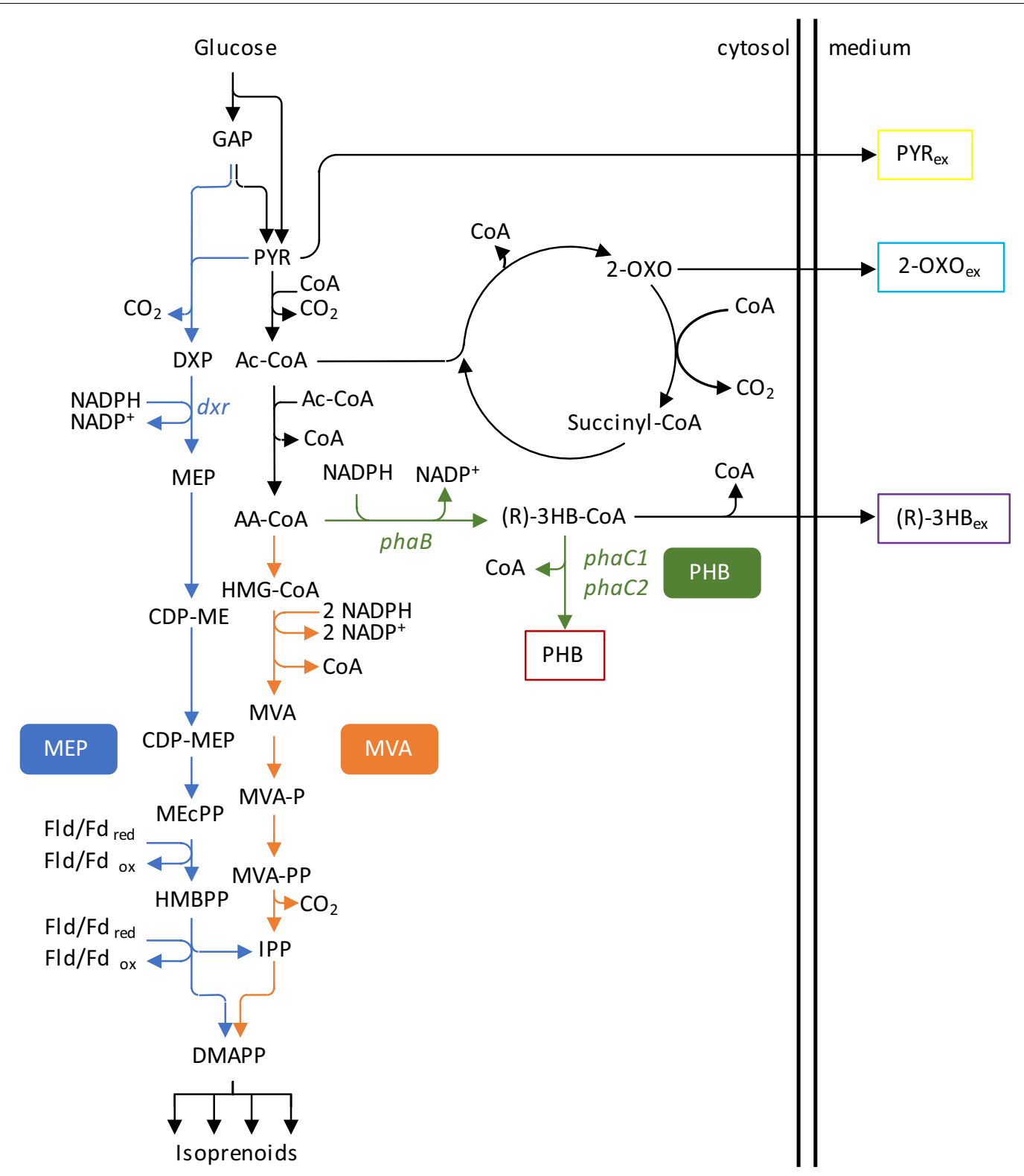

Fig. 1 Network investigated in this work. The metabolic map shows the steps involved in the biochemical conversion of glucose to isoprenoids. Isoprenoid biosynthesis can occur via the two orthogonal 2-C-methyl-D-erythritol 4-phosphate (MEP, blue arrows) and mevalonate (MVA, orange arrows) pathways. For the MEP pathway, the 1-deoxy-D-xylulose 5-phosphate reductoisomerase ( $d x r$ ) gene is shown. This gene is targeted for inactivating the endogenous isoprenoid pathway. Both modules branch from the central metabolism and converge to isopentenyl-diphosphate (IPP) and dimethylallyl-diphosphate (DMAPP), which are the precursors of all isoprenoids. Moreover, the biosynthetic pathway of the storage compound poly- $\beta$-hydroxybutyrate (PHB) is included (box with red outline). This consists of two enzymatic reactions encoded by the genes phaB and phaC1 and phaC2 (both in green). The network includes also the schematic representation of the Krebs cycle, which includes conversion of 2-oxoglutarate (2-OXO) to succinyl-CoA. Eventually, accumulation of PYR or 2-OXO can result in their secretion in the medium (boxes with yellow and light-blue outline, respectively). Additionally, conversion of (R)-3hydroxybturyryl-CoA ((R)-3HB-CoA) to (R)-3HB can result in the secretion of the latter compound (box with purple outline). Other abbreviations: reduced flavodoxin (Fld), ferredoxin (Fd), red: reduced, ox: oxidized. GAP (glyceraldehyde-3-phosphate), PYR (pyruvate), CDP-ME (4-(cytidine 5'-diphospho)-2-C-methyl-D-erythritol), CDP-MEP (2-phospho-4-(cytidine 5'-dip hospho)-2-C-methyl-D-erythritol), MECPP (2-C-methyl-D-erythritol 2,4-cyclodiphosphate), Ac-CoA (acetyl-CoA), AA-CoA (acetoacetyl-CoA), HMG-CoA (S)-3-hydroxy-3-methylglutaryl-CoA), MVA-P ((R)-5-phosphomevalonate), MVA-PP ((R)-5-diphosphomevalonate) 
pathway compartmentalized in the chloroplasts and the MVA pathway expressed in the cytosol [9]. Since the first implementation of a heterologous MVA pathway in Escherichia coli [10], the number of studies focusing on the synthesis of isoprenoids via microbial cell factories has increased a lot. Efforts for improving bioproduction have been focusing either on engineering of the endogenous MEP pathway [11-13], or by co-expressing the heterologous MVA counterpart [10,14, 15]. Moreover, to limit the effect of unfavourable regulatory control of the endogenous pathway, substitution by an orthogonal isoprenoid pathway has been reported [16].

In microorganisms, isoprenoids are often membranebound molecules-like carotenoids, ubiquinones, chlorophylls and sterols - which are indispensable for growth $[17,18]$. Therefore, during a batch cultivation, their volumetric concentration is expected to increase as consequence of microbial growth inside the reactor. Such type of metabolism for isoprenoid biosynthesis is defined as growth-coupled. For strain improvement purposes, growth-coupled production has been largely employed. In such a scenario, the product of interest becomes a mandatory by-product of growth, and therefore microbial growth becomes the driving force of production [19]. This production-growth association has already been exploited for enhancing isoprenoid biosynthesis by laboratory evolution [20].

Nevertheless, growth-coupled production contains an inherent trade-off between substrate use for (i) biomass production and maintenance, and (ii) product formation [21]. Thus, if biomass formation is prevented, in principle more substrate is available for product synthesis.

Microbial metabolism has been engineered to produce non-native isoprenoid molecules as pharmaceuticals, perfumes, food flavourings and biofuels [1-6]. These compounds are not required for growth, and often excreted. Coupling of product formation to microbial growth is therefore not a necessity, and growth-uncoupled production would be an advantageous option.

Apart from $\mathrm{C}, \mathrm{H}$ and $\mathrm{O}$, isoprenoids do not contain other biomass-specific elements like $\mathrm{P}, \mathrm{S}$ or N. Therefore, under $\mathrm{P}, \mathrm{S}$ or N-limited incubation conditions, glucose can in principle be converted into isoprenoid but not into microbial biomass. Improved isoprenoid/biomass ratios have already been obtained by using nutrient-limited culturing conditions [22-25]. Nonetheless, as a highly regulated primary metabolism, isoprenoid biosynthesis has been largely optimized for growth-coupled production, and decoupling isoprenoid synthesis from microbial growth has been overlooked as a production strategy.

In a recent study, the concept of redesigning biosynthetic networks based on orthogonality principles was introduced [26]. This idea entails that a non-native metabolic route that minimizes the interaction with the endogenous biomass-producing pathways can be exploited for bioproduction. Intrinsic independence exists between the two isoprenoid pathways MEP and MVA. Therefore, regulatory control affecting the MEP pathway should not affect the MVA pathway, and vice versa. Moreover, in a recent study, functional replacement of the native MEP with the heterologous MVA pathway was described in the bacterium Rhodobacter sphaeroides [16], a microbial platform organism that is gaining interest for isoprenoid biosynthesis. This organism is able to synthesize intracellular membranes which can accommodate isoprenoids such as carotenoids and bacteriochlorophylls. Moreover, it is also a natural producer of coenzyme $Q_{10}$. Apart from these native isoprenoid molecules, heterologous production of lycopene [27] and sesquiterpenes [28] has also been reported in this species.

Growth and isoprenoid synthesis in $R$. sphaeroides has been studied using defined medium, where the introduction of the heterologous MVA pathway revealed potential for growth-independent isoprenoid biosynthesis [29]. Under these conditions, the storage compound poly- $\beta$ hydroxybutyrate (PHB) was also accumulated [29]. Additionally, a mutual stimulating effect between the MEP and MVA pathways has been observed [30].

In this study, we investigate the behaviour of the two isoprenoid pathways for amorphadiene production in $R$. sphaeroides during different growth modes, as well as their interaction with the pathway for the carbon- and energy reserve material poly- $\beta$-hydroxybutyrate, PHB. By means of ${ }^{13} \mathrm{C}$ metabolic flux ratio analysis, we assess the effect of genetic modifications (i.e. for elimination of PHB accumulation) and environmental changes (nitrogen limitation) on isoprenoid pathways capacities. Ultimately, we demonstrate that exclusive use of the orthogonal MVA pathway in combination with elimination of PHB synthesis is a promising strategy for attaining growth-independent production of isoprenoids.

\section{Materials and methods \\ Strains and standard cultivation conditions}

The strains and plasmids used in this study are listed in Tables 1 and 2, respectively. The Rs265_AphaC1 1 phaC2 strain was kindly donated by Isobionics BV. Preculturing of $R$. sphaeroides was performed in 250-mL Erlenmeyer flasks containing $25 \mathrm{~mL}$ of modified Sistrom's minimal medium (SMM). As previously described [30], the medium contained glucose $(3.0 \mathrm{~g} / \mathrm{L})$ as carbon source, and $\mathrm{NH}_{4} \mathrm{Cl}(1.0 \mathrm{~g} / \mathrm{L})$ as nitrogen source. Moreover, the SMM contained (per litre): $3.48 \mathrm{~g} \mathrm{KH}_{2} \mathrm{PO}_{4}$, $0.1 \mathrm{~g}$ glutamic acid, $0.04 \mathrm{~g}$ L-aspartic acid, $0.5 \mathrm{~g} \mathrm{NaCl}$, $0.02 \mathrm{~g}$ nitrilotriacetic acid, $0.3 \mathrm{~g} \mathrm{MgSO}_{4} \cdot 7 \mathrm{H}_{2} \mathrm{O}, 0.0334 \mathrm{~g}$ 
Table 1 Strains used in this study

\begin{tabular}{|c|c|c|}
\hline Strain & Description & Source of reference \\
\hline \multicolumn{3}{|l|}{ E. coli } \\
\hline S17-1 & Host strain for conjugation, thi pro recA hsdR [RP4-2Tc::Mu-Km::Tn7] $\mathrm{Tp}^{r} \mathrm{Sm}^{r}$ & Laboratory stock \\
\hline \multicolumn{3}{|l|}{ R. sphaeroides } \\
\hline Rs265 & Wild-type & $\begin{array}{l}\text { Derivative of } \\
\text { ATCC } 35035 \text {, Isobi- } \\
\text { onics BV }\end{array}$ \\
\hline Rs265_sphaC14phaC2 & Rs265 with the PHB biosynthetic pathway knocked-out via phaC1 and phaC2 deletion & Isobionics BV \\
\hline Rs265_4phaB & Rs265 with the PHB biosynthetic pathway knocked-out via phaB deletion & {$[31]$} \\
\hline Rs265-MVA_ $\Delta d x r$ & $\begin{array}{l}\text { Rs265 with chromosomally integrated the MVA pathway operon and knocked-out the endogenous } \\
\text { MEP pathway via dxr deletion }\end{array}$ & {$[16]$} \\
\hline Rs265-MVA_.ddxr $\Delta p h a B$ & $\begin{array}{l}\text { Rs } 265 \text { with chromosomally integrated the MVA pathway operon and knocked-out the endogenous } \\
\text { MEP (via } d x \text { r deletion) and PHB (via phaB deletion) biosynthetic pathways }\end{array}$ & This study \\
\hline
\end{tabular}

Table 2 Plasmids used in this study

\begin{tabular}{ll}
\hline Plasmid & Description \\
\hline pBBR-ads & pBBR1MCS-2+crtE promoter and ads (amorphadiene synthase) \\
of reference
\end{tabular}

$\mathrm{CaCl}_{2} \cdot 2 \mathrm{H}_{2} \mathrm{O}, \quad 0.002 \mathrm{~g} \quad \mathrm{FeSO}_{4} \cdot 7 \mathrm{H}_{2} \mathrm{O}$, and $0.0002 \mathrm{~g}$ $\left(\mathrm{NH}_{4}\right)_{6} \mathrm{Mo}_{7} \mathrm{O}_{24}$. Trace elements were added $(0.01 \% \mathrm{v} / \mathrm{v})$ from a stock solution containing: $17.65 \mathrm{~g} / \mathrm{L}$ disodium EDTA, $109.5 \mathrm{~g} / \mathrm{L} \mathrm{ZnSO}_{4} \cdot 7 \mathrm{H}_{2} \mathrm{O}, 50 \mathrm{~g} / \mathrm{L} \mathrm{FeSO}_{4} \cdot 7 \mathrm{H}_{2} \mathrm{O}$, $15.4 \mathrm{~g} / \mathrm{L} \mathrm{MnSO}_{4} \cdot 7 \mathrm{H}_{2} \mathrm{O}, 3.92 \mathrm{~g} / \mathrm{L} \mathrm{CuSO}_{4} \cdot 5 \mathrm{H}_{2} \mathrm{O}, 2.48 \mathrm{~g} / \mathrm{L}$ $\mathrm{Co}\left(\mathrm{NO}_{3}\right)_{2} \cdot 6 \mathrm{H}_{2} \mathrm{O}$, and $0.0114 \mathrm{~g} / \mathrm{L} \mathrm{H}_{3} \mathrm{BO}_{3}$. Vitamins were added $(0.01 \% \mathrm{v} / \mathrm{v})$ from a stock containing: $10 \mathrm{~g} / \mathrm{L}$ nicotinic acid, $5 \mathrm{~g} / \mathrm{L}$ thiamine $\mathrm{HCl}$, and $0.1 \mathrm{~g} / \mathrm{L}$ biotin.

\section{Generation of double-KO strain via CRISPR-Cas9 counter-selection}

The primers used in this study are listed in Additional file 1: Table S1. Deletion of phaB was performed as previously described [31], using the pBBR_Cas9_ $\Delta p h a B_{-}$ HR plasmid. Such plasmid was transferred from E. coli S17 cells to Rs265-MVA_ $\Delta d x r$ via diparental conjugation, resulting in the double mutant strain Rs265-MVA_ $\Delta d x r \Delta p h a B$. By employing the pBBR Cas9_ $\triangle p h a B \_H R$ plasmid, phaB could be removed by homologous-recombination, and Cas9-based counterselection of cells with intact genomic copies of phaB.

\section{Diparental conjugation of $R$. sphaeroides}

Diparental conjugation for transferring amorphadiene producing plasmids was performed as previously described [30] using RÄ medium. Such medium contained, per litre: $3 \mathrm{~g}$ malic acid, $0.2 \mathrm{~g} \mathrm{MgSO}_{4} \cdot 7 \mathrm{H}_{2} \mathrm{O}, 1.2 \mathrm{~g}$
$\left(\mathrm{NH}_{4}\right)_{2} \mathrm{SO}_{4}, 0.07 \mathrm{~g} \mathrm{CaCl}_{2} \cdot 2 \mathrm{H}_{2} \mathrm{O}, 1.5 \mathrm{~mL}$ of microelements stock solution, $2 \mathrm{~mL}$ of vitamin stock solution and $5 \mathrm{~mL}$ of phosphate buffer. In case of R $\ddot{A}$ agar medium, $15 \mathrm{~g} / \mathrm{L}$ agar was added. The microelements solution contained: $0.5 \mathrm{~g} / \mathrm{L} \mathrm{Fe}(\mathrm{II})$-Citrate, $0.02 \mathrm{~g} / \mathrm{L} \mathrm{MnCl}_{2} \cdot 4 \mathrm{H}_{2} \mathrm{O}, 0.005 \mathrm{~g} / \mathrm{L}$ $\mathrm{ZnCl}_{2}, 0.0025 \mathrm{~g} / \mathrm{L} \mathrm{KBr}, 0.0025 \mathrm{~g} / \mathrm{L} \mathrm{KI}, 0.0023 \mathrm{~g} / \mathrm{L}$ $\mathrm{CuSO}_{4} \cdot 5 \mathrm{H}_{2} \mathrm{O}, 0.041 \mathrm{~g} / \mathrm{L} \mathrm{Na}_{2} \mathrm{MoO}, 0.005 \mathrm{~g} / \mathrm{L} \mathrm{CoCl} \mathrm{Cl}_{2} \cdot 6 \mathrm{H}_{2} \mathrm{O}$, $0.0005 \mathrm{~g} / \mathrm{L} \quad \mathrm{SnCl}_{2} \cdot 2 \mathrm{H}_{2} \mathrm{O}, 0.0006 \mathrm{~g} / \mathrm{L} \quad \mathrm{BaCl}_{2} \cdot 2 \mathrm{H}_{2} \mathrm{O}$, $0.031 \mathrm{~g} / \mathrm{L} \mathrm{AlCl}, 0.41 \mathrm{~g} / \mathrm{L} \mathrm{H}_{3} \mathrm{BO}_{3}, 0.02 \mathrm{~g} / \mathrm{L}$ EDTA. The vitamin solution contained: $0.2 \mathrm{~g} / \mathrm{L}$ nicotinic acid, $0.4 \mathrm{~g} / \mathrm{L}$ thiamine $\mathrm{HCl}, 0.008 \mathrm{~g} / \mathrm{L}$ biotin, $0.2 \mathrm{~g} / \mathrm{L}$ nicotinamide. The phosphate buffer contained $0.6 \mathrm{~g} / \mathrm{L} \mathrm{KH}_{2} \mathrm{PO}_{4}$ and $0.9 \mathrm{~g} / \mathrm{L}$ $\mathrm{K}_{2} \mathrm{HPO}_{4}$.

\section{Cultivation in nitrogen excess and nitrogen-limited conditions}

After overnight preculturing on SMM, R. sphaeroides cultures were transferred to fresh SMM with a starting $\mathrm{OD}_{600}$ of 0.1 , and incubated at $30{ }^{\circ} \mathrm{C}$ with $250 \mathrm{rpm}$. Cultivations were performed for biological triplicates in $250-\mathrm{mL}$ Erlenmeyer flasks, each filled with $45 \mathrm{~mL}$ of SMM medium and $5 \mathrm{~mL}$ of filter-sterilized dodecane. SMM composition differed between nitrogen excess condition and nitrogen-limited conditions only in the initial $\mathrm{NH}_{4} \mathrm{Cl}$ concentration: $1.0 \mathrm{~g} / \mathrm{L}$ and $0.25 \mathrm{~g} / \mathrm{L}$, respectively. Initial glucose concentration remained $3.0 \mathrm{~g} / \mathrm{L}$ in both cases. Amorphadiene titers were measured after glucose 
depletion. This occurred after $24 \mathrm{~h}$ (for nitrogen excess condition) or after $48 \mathrm{~h}$ (for nitrogen-limited condition). At the same time, the content of the flasks was harvested by centrifugation and further processed for analytical measurements.

${ }^{13} \mathrm{C}$-metabolic flux ratio analysis of isoprenoid biosynthesis Isoprenoid flux ratios analyses were performed as previously described [30] in 10-mL Erlenmeyer flasks containing $1.8 \mathrm{~mL}$ of labelled SMM medium and $0.2 \mathrm{~mL}$ of filter-sterilized dodecane. $\left[1-{ }^{13} \mathrm{C}\right]-$ and $\left[4-{ }^{13} \mathrm{C}\right]$-glucose tracers had an initial concentration of $3.0 \mathrm{~g} / \mathrm{L}$. For nitrogen excess and limited conditions, the initial $\mathrm{NH}_{4} \mathrm{Cl}$ concentration used was the same as for the cultivations in 250-mL flasks: 1.0 and $0.25 \mathrm{~g} / \mathrm{L}$, respectively. Samples for GC-MS measurement were taken at glucose depletion. MEP and MVA pathways capacities were obtained by multiplying the flux ratios determined via GC-MS with the amorphadiene titers measured via GC-FID for the 250-mL cultivations.

\section{Cultivation under resting cell conditions}

For resting cell cultivations, exponentially growing cells on SMM with nitrogen excess conditions were incubated until mid-exponential phase. Then, cells were pelleted by centrifugation at $4255 \mathrm{~g}$ for $10 \mathrm{~min}$ at room temperature. Subsequently, pellets were washed twice with sterile physiologic solution $(\mathrm{NaCl} 9 \mathrm{~g} / \mathrm{L})$ and centrifuged at $4255 \mathrm{~g}$ for $5 \mathrm{~min}$. Washed pellets were inoculated with a starting $\mathrm{OD}_{600}$ of 1.0 on 'nitrogen-free SMM' containing $5.0 \mathrm{~g} / \mathrm{L}$ glucose and $0.0 \mathrm{~g} / \mathrm{L} \mathrm{NH}_{4} \mathrm{Cl}$. Initial biomass concentration was determined at the moment of the inoculum with a sample of $5 \mathrm{~mL}$ for TOC-L analysis. Then, incubation proceeded at $30^{\circ} \mathrm{C}$ with $250 \mathrm{rpm}$. Samples for $\mathrm{OD}_{600}, \mathrm{pH}$ and amorphadiene measurements were taken at a regular interval until the $\mathrm{pH}$ dropped below 5.5. Determination of productivity and yields were performed for samples taken within the first $24 \mathrm{~h}$.

\section{Analytics}

Cell density was monitored by measuring the optical density at $600 \mathrm{~nm}\left(\mathrm{OD}_{600}\right)$. Amorphadiene concentration was measured via GC-FID as previously described [29]. Glucose, PHB and organic acid concentrations were determined via (U)HPLC as previously described [29]. For pyruvate and crotonic acid (resulting from PHB hydrolysis) identification, DAD detector was used, while for glucose, 2-oxoglutarate and 3-hydroxybutyrate determination, the RID detector was used. Determination of biomass concentration via TOC-L was performed by measuring the nitrogen content of the pellet, which was then used to calculate the active biomass concentration using the elemental composition of $R$. sphaeroides of $\mathrm{CH}_{1.99} \mathrm{O}_{0.5} \mathrm{~N}_{0.19}$ [29]. Identification of unknown compounds in the spent medium was obtained via ${ }^{1} \mathrm{H}$-nuclear magnetic resonance spectroscopy $\left({ }^{1} \mathrm{H}-\mathrm{NMR}\right)$ measurements performed in $\mathrm{D}_{2} \mathrm{O}$ on a Bruker Avance III $400 \mathrm{MHz}$ NMR spectrometer.

\section{Results}

\section{Prevention of PHB formation and its effect}

\section{on amorphadiene biosynthesis}

Culturing $R$. sphaeroides under nitrogen-limited conditions could theoretically result in growth-independent isoprenoid synthesis via the native MEP and the heterologous MVA pathways. However, upon consumption of the limited available nitrogen, $R$. sphaeroides stores excess carbon intracellularly as PHB, a nitrogen-free carbon and energy storage compound [29]. Aiming to increase isoprenoid production, we reasoned that deletion of one of the PHB synthesis genes would block PHB production under nitrogen-limited conditions and could therefore increase the flux through the MVA pathway. Deletion of the phaC1 and phaC2 genes, that code for the PHB polymerase, is the established approach for eliminating PHB biosynthesis in R. sphaeroides [32-35]. Nonetheless, this does not prevent activity of the NADPH-dependent acetoacetyl-CoA reductase $\mathrm{PhaB}$, which could result in the undesired accumulation of 3-hydroxybutyryl-CoA or excretion of 3-hydroxybutyrate (Fig. 1). In a recent study, we demonstrated that deletion of the phaB gene prevents PHB biosynthesis [31]. Here, we confirmed that the deletion of either the phaB gene (Rs265_ $\triangle p h a B$ strain) or the combined deletion of the phaC1 and phaC2 genes (Rs265__phaC1 1 phaC2 strain) prevents PHB formation both under nitrogen excess and nitrogen-limited conditions (Fig. 2a). As observed before [29], the wild-type (Rs265) strain produced substantial amounts of PHB, especially under nitrogen-limiting conditions.

The pBBR-ads plasmid harbouring the heterologous amorphadiene synthase gene was transferred to the various $R$. sphaeroides strains by conjugation. The resulting strains were cultured under both nitrogen excess and nitrogen-limited conditions. At glucose depletion, we determined (Table 3): amorphadiene titers $\left(C_{P}\right)$, yield of amorphadiene on glucose $\left(Y_{P / S}\right)$, active biomass concentration $\left(C_{X}\right)$ and the amorphadiene on biomass ratio $\left(Y_{P / X}\right)$. We observed that, for the Rs265_LphaB:pBBRads and Rs265_AphaC1 1 phaC2:pBBR-ads strains that use only the endogenous MEP pathway (MEP-only strains), elimination of PHB synthesis does not result in higher amorphadiene/biomass ratios compared to the Rs265 wt strain (Fig. 2b, Table 3). In fact, these ratios remained unaffected when moving from nitrogen excess to nitrogen-limited conditions (Table 3 ) at a value of $2.9 \pm 0.2 \mathrm{mg} \cdot \mathrm{g}$ of biomass ${ }^{-1}$. Interestingly, the 


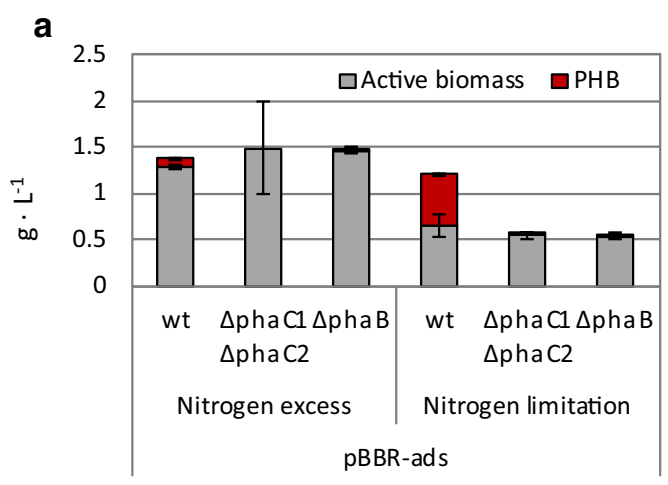

C

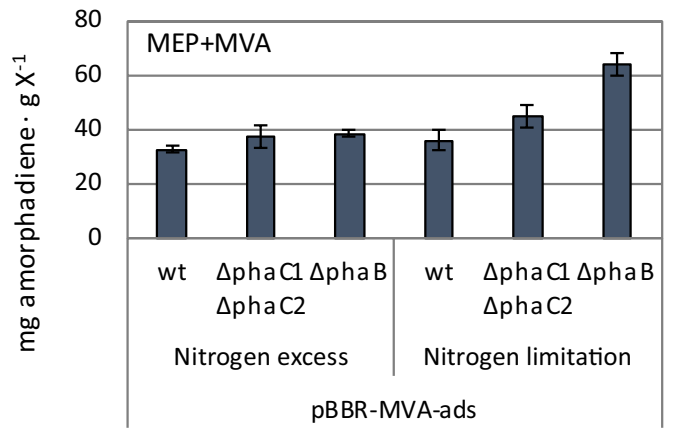

e

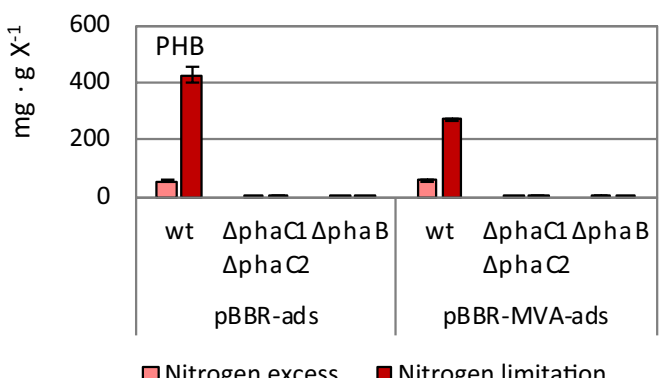

g

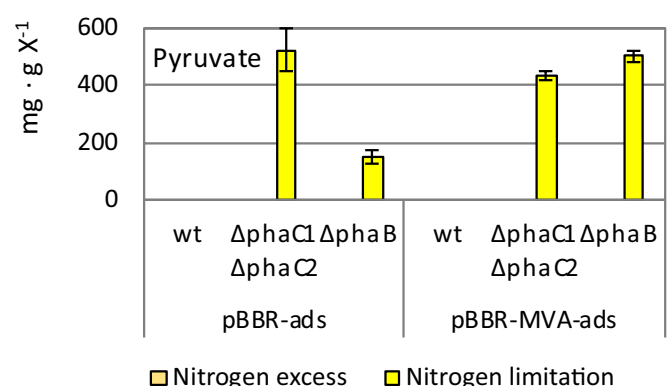

b

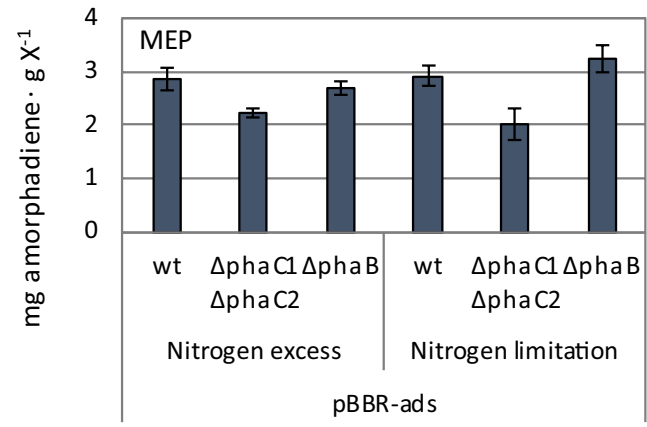

d

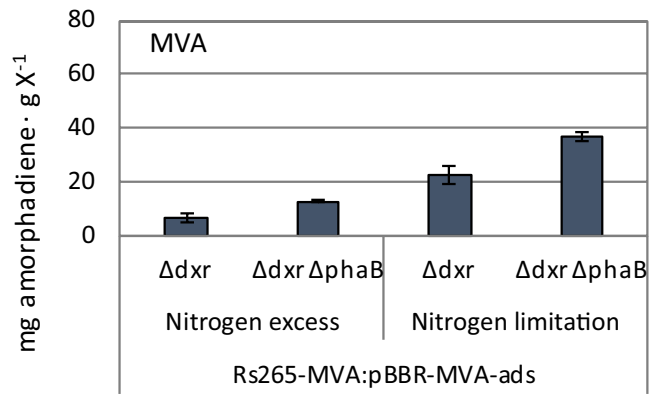

f

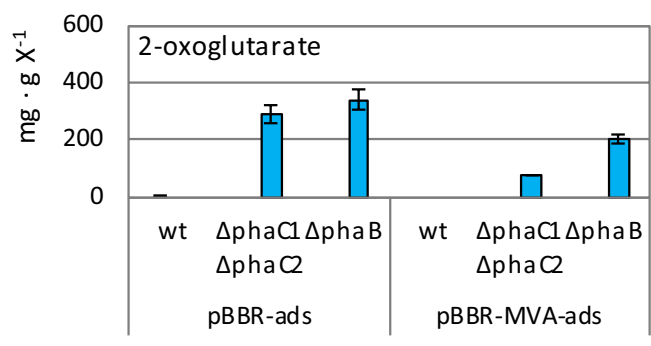

$\square$ Nitrogen excess $\square$ Nitrogen limitation

h

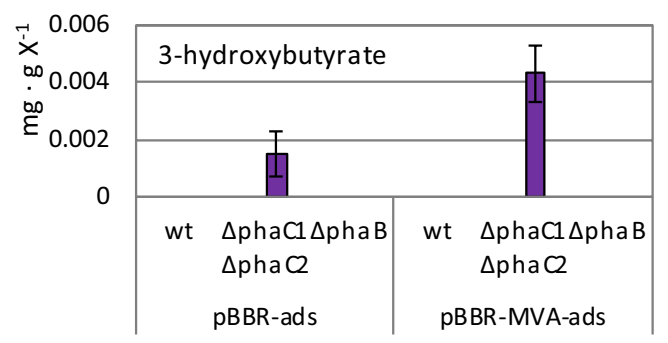

$\square$ Nitrogen excess $\square$ Nitrogen limitation

Fig. 2 Deletion of PHB pathway and effect on amorphadiene yield on biomass. a Effect of initial medium C/N ratio on active biomass and PHB concentrations. b-d mg amorphadiene $\mathrm{g}$ of biomass ${ }^{-1}$ for wt, $\triangle$ phaC1 $\triangle$ phaC2 and $\triangle p h a B$ background in strains relying on $\mathbf{b}$ only MEP pathway (pBBR-ads), c MEP and MVA pathway (pBBR-MVA-ads), $\mathbf{d}$ only MVA pathway (Rs265-MVA:pBBR-MVA-ads strain). e, $\mathbf{f}$ mg $\mathrm{g}$ of biomass ${ }^{-1} \mathrm{calculated} \mathrm{for}^{-1}$ e PHB, $\mathbf{f}$ 2-oxoglutarate, $\mathbf{g}$ pyruvate and $\mathbf{h}$ 3-hydroxybutyrate during nitrogen excess and nitrogen limited conditions 
Table 3 Titers and yields obtained from shaking flasks experiments of strains Rs265 and Rs265-MVA

\begin{tabular}{|c|c|c|c|c|c|c|c|c|c|c|}
\hline \multirow[t]{2}{*}{ Plasmid } & \multirow[t]{2}{*}{ Condition } & \multirow[t]{2}{*}{ Genomic background } & \multicolumn{2}{|c|}{$\begin{array}{l}\text { Amorphadiene } \\
\text { titers, } C_{P}\left(\mathrm{mg} \cdot \mathrm{L}^{-1}\right)\end{array}$} & \multicolumn{2}{|c|}{$\begin{array}{l}\text { Amorphadiene } \\
\text { yield on glucose, } \\
Y_{P / S}\left(\mathrm{mg} \cdot \mathrm{g}^{-1}\right) \\
\end{array}$} & \multicolumn{2}{|c|}{$\begin{array}{l}\text { Final Biomass } \\
\text { concentration, } C_{X} \\
\left(\mathrm{~g} \cdot \mathrm{L}^{-1}\right)\end{array}$} & \multicolumn{2}{|c|}{$\begin{array}{l}\text { Amorphadiene } \\
\text { on Biomass, } Y_{P / X} \\
\left(\mathrm{mg} \cdot \mathrm{g}^{-1}\right)\end{array}$} \\
\hline & & & Value & St. dev. & Value & St. dev. & Value & St. dev. & Value & St. dev. \\
\hline \multirow[t]{6}{*}{ pBBR-ads } & Nitrogen excess & $w t$ & 4.4 & 0.3 & 1.41 & 0.15 & 1.533 & 0.062 & 2.9 & 0.2 \\
\hline & & $\Delta p h a C 1 \Delta p h a C 2$ & 3.3 & 0.1 & ND & ND & 1.491 & 0.000 & 2.2 & 0.1 \\
\hline & & $\triangle p h a B$ & 4.7 & 0.2 & 1.49 & 0.07 & 1.724 & 0.110 & 2.7 & 0.1 \\
\hline & Nitrogen limited & $w t$ & 2.4 & 0.2 & 0.73 & 0.05 & 0.805 & 0.122 & 2.9 & 0.2 \\
\hline & & $\Delta p h a C 1 \Delta p h a C 2$ & 1.4 & 0.2 & ND. & ND. & 0.693 & 0.034 & 2.0 & 0.3 \\
\hline & & $\triangle p h a B$ & 2.2 & 0.2 & 0.69 & 0.04 & 0.693 & 0.112 & 3.2 & 0.2 \\
\hline \multirow[t]{6}{*}{ pBBR-MVA-ads } & Nitrogen excess & $w t$ & 41.8 & 1.8 & 13.64 & 0.56 & 1.289 & 0.019 & 32.4 & 1.4 \\
\hline & & $\triangle p h a C 1 \triangle p h a C 2$ & 39.3 & 4.4 & ND. & ND. & 1.060 & 0.856 & 37.1 & 4.2 \\
\hline & & $\triangle p h a B$ & 56.4 & 2.1 & 18.25 & 0.68 & 1.466 & 0.036 & 38.5 & 1.5 \\
\hline & Nitrogen limited & $w t$ & 23.6 & 2.3 & 7.65 & 0.76 & 0.656 & 0.115 & 35.9 & 3.6 \\
\hline & & $\Delta p h a C 1 \Delta p h a C 2$ & 24.1 & 2.1 & ND. & ND. & 0.539 & 0.260 & 44.8 & 3.9 \\
\hline & & $\triangle p h a B$ & 34.4 & 2.2 & 11.38 & 0.73 & 0.539 & 0.022 & 63.7 & 4.0 \\
\hline \multirow[t]{4}{*}{ pBBR-MVA-ads } & Nitrogen excess & MVAint_ddxr & 15.6 & 3.9 & 3.41 & 0.23 & 2.229 & 0.144 & 7.0 & 1.7 \\
\hline & & MVAint_sdxrsphaB & 28.0 & 1.0 & 9.67 & 0.86 & 2.187 & 0.080 & 12.8 & 0.5 \\
\hline & Nitrogen limited & MVAint_ddxr & 13.5 & 1.9 & 3.80 & 0.82 & 0.597 & 0.142 & 22.6 & 3.1 \\
\hline & & MVAint_sdxr $\Delta p h a B$ & 19.5 & 0.8 & 5.65 & 0.29 & 0.528 & 0.161 & 36.9 & 1.6 \\
\hline
\end{tabular}

Data were obtained from end-point measurements under nitrogen excess and nitrogen-limited conditions

ND not determined

Rs265_sphaC1 1 phaC2:pBBR-ads strain showed an even lower amorphadiene/biomass ratio compared to the Rs265:pBBR-ads and the Rs265_dphaB:pBBR-ads strains (Table 3). In summary, when only the endogenous MEP pathway was active, isoprenoid biosynthesis appeared to be strictly growth-coupled. Moreover, the MEP flux was insensitive or negatively affected by the impaired PHB synthesis.

We subsequently transformed the available Rs265,

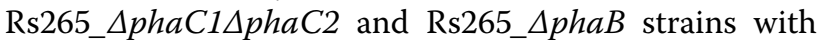
the orthogonal MVA pathway, cloned in the pBBR-MVAads plasmid. The amorphadiene/biomass ratio increased 10- to 20-fold for all the strains tested (Fig. 2c, Table 3). The highest increase was observed for the $\triangle p h a B$ strain (Rs265_LphaB:pBBR-MVA-ads), reaching a ratio of $63.7 \pm 4.0 \mathrm{mg} \cdot \mathrm{g}$ of biomass $^{-1}$ (Fig. 2c, Table 3). This value was significantly higher than the ratio reached by the strain with a functional PHB synthesis (Rs265:pBBRMVA-ads), which was $35.9 \pm 3.6 \mathrm{mg} \cdot \mathrm{g}$ of biomass $^{-1}$ (Fig. 2c, Table 3).

Increase of the MVA pathway flux, as consequence of the phaB deletion, was confirmed also for the Rs265MVA_ $\Delta d x r$ strain, for which the MEP pathway is inactivated via the deletion of the 1-deoxy-D-xylulose 5 -phosphate reductoisomerase $(d x r)$ gene, after genomic integration of the MVA pathway (Fig. 1). This strain relies exclusively on the non-native isoprenoid route
(MVA-only) [16]. Also here, a substantial increase in the amorphadiene/biomass ratio was observed during both nitrogen excess and nitrogen-limited conditions (Fig. 2d, Table 3). The highest value observed was for the Rs265-MVA_ $\Delta d x r \Delta p h a B: p B B R-M V A-a d s$ strain, with $36.9 \pm 1.6 \mathrm{mg} \cdot \mathrm{g}$ of biomass ${ }^{-1}$ during nitrogen limitation.

In summary, although both the $\Delta p h a C 1 \Delta p h a C 2$ and $\triangle p h a B$ knockouts were equally effective in reducing $\mathrm{PHB}$ synthesis, the $\triangle p h a B$ knockout strain produced more amorphadiene, both volumetrically and per biomass unit (Table 3).

\section{Organic acids secretion as consequence of PHB deletion}

We reasoned that comparing the secretion profiles between $\triangle p h a B$ and $\triangle p h a C 1 \Delta p h a C 2$ could provide additional insights on the beneficial effect of $\triangle p h a B$ on isoprenoid synthesis. Therefore, we quantified by HLPC analysis the organic acids in the spent medium of Rs265,

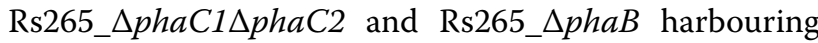
either pBBR-ads or pBBR-MVA-ads plasmids (Fig. 2e-h). For both $\triangle p h a B$ and $\triangle p h a C 1 \Delta p h a C 2$ strains, 2-oxoglutarate ( 80 to $340 \mathrm{mg} \cdot \mathrm{g}$ of biomass ${ }^{-1}$, Fig. 2f) and pyruvate $\left(150\right.$ to $500 \mathrm{mg} \cdot \mathrm{g}$ of biomass ${ }^{-1}$, Fig. $2 \mathrm{~g}$ ) were the main by-products. Both compounds require coenzymeA (CoA) for proceeding further in the metabolism via oxidative decarboxylation (Fig. 1). Excretion of these 
compounds suggests that free $\mathrm{CoA}$ is limiting when $\mathrm{PHB}$ biosynthesis is prevented.

The $\Delta p h a C 1 \Delta p h a C 2$ strains secreted an additional unknown compound, which was identified as 3-hydroxybutyrate (3HB) by NMR (Additional file 1: Fig. S1). The spent medium for the Rs265_sphaC1 $\Delta$ phaC2:pBBRads strain showed a value of $0.002 \pm 0.001 \mathrm{mg}$ $3 \mathrm{HB} \cdot \mathrm{g}$ of biomass ${ }^{-1}$ (Fig. 2h). In contrast, a value of $0.004 \pm 0.001 \mathrm{mg} 3 \mathrm{HB} \cdot \mathrm{g}$ of biomass ${ }^{-1}$ was observed

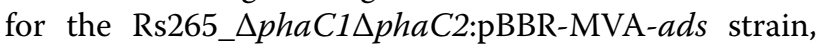
which expressed the heterologous MVA pathway (Fig. 2h). Therefore, under nitrogen limitation and upon expression of the MVA pathway, the amount of $3 \mathrm{HB}$ secreted increased significantly compared to when only the MEP pathway was active.

\section{${ }^{13} \mathrm{C}$ metabolic flux ratio analysis of isoprenoid biosynthesis under different growth conditions}

The Rs265__phaB:pBBR-MVA-ads strain, that overexpresses the MVA pathway and still has an active MEP pathway, showed the highest amorphadiene/biomass ratio under nitrogen-limited conditions (Fig. 2c, Table 3). Additionally, we observed that, independent from the cultivation conditions and the presence of an active PHB synthesis pathway, the dual-pathway (co-expressing MEP and MVA pathways) strains largely outperformed the single-pathway strains (Fig. 2b-d, Table 3). We therefore decided to further investigate the separate and combined contribution of the isoprenoid pathways to the amorphadiene production by ${ }^{13} \mathrm{C}$ flux ratio analysis.

MEP and MVA pathways are known to exert a reciprocal stimulation [30,36]. To better understand their mode of interaction under the conditions tested, we determined their contribution via ${ }^{13} \mathrm{C}$ metabolic flux ratio analysis of the Rs265:pBBR-MVA-ads and Rs265__phaB:pBBRMVA-ads strains. We therefore compared the resulting amorphadiene/biomass ratios for each pathway with the ones determined for (i) the Rs265:pBBR-ads and Rs265_sphaB:pBBR-ads (MEP-only) strains, (ii) the Rs265-MVA_ $\Delta d x r$ :pBBR-MVA-ads and (iii) Rs265MVA_ $\Delta d x r \Delta p h a B: p B B R-M V A-a d s$ (MVA-only) strains. Previously, this ${ }^{13} \mathrm{C}$-method provided important insights on the flux ratios upon co-expression of the MEP and MVA pathways [30]. Under nitrogen excess condition, we observed that the dual-pathway strains with active MEP and MVA pathways showed a higher amorphadiene/biomass ratio for each isoprenoid route compared to when these were active individually (Fig. 3a). We therefore confirmed that, during nitrogen excess conditions, co-expression of the two isoprenoid pathways resulted in enhancement of their capacities in the Rs265 and Rs265_ $\triangle p h a B$ strains harbouring the pBBR-MVA-ads a

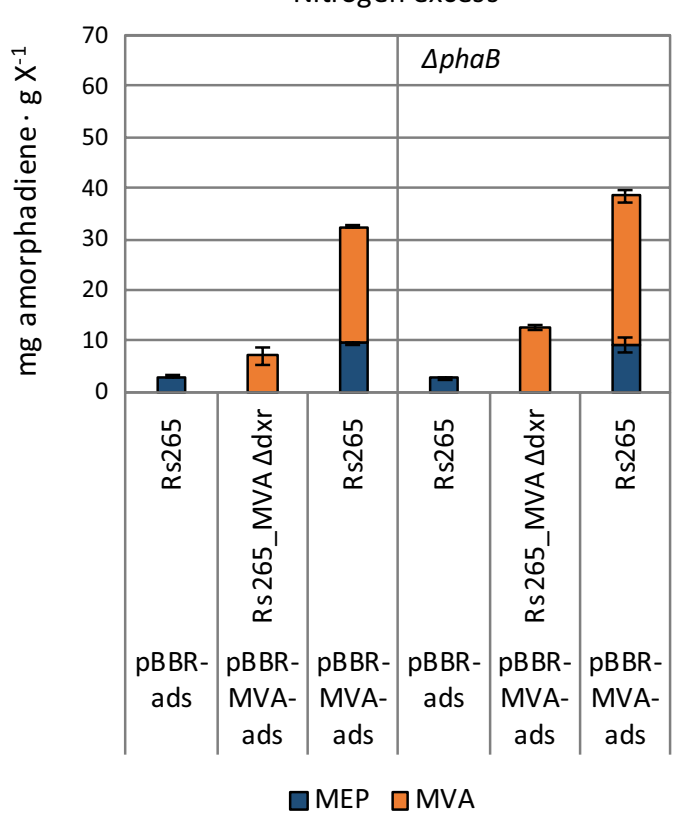

b

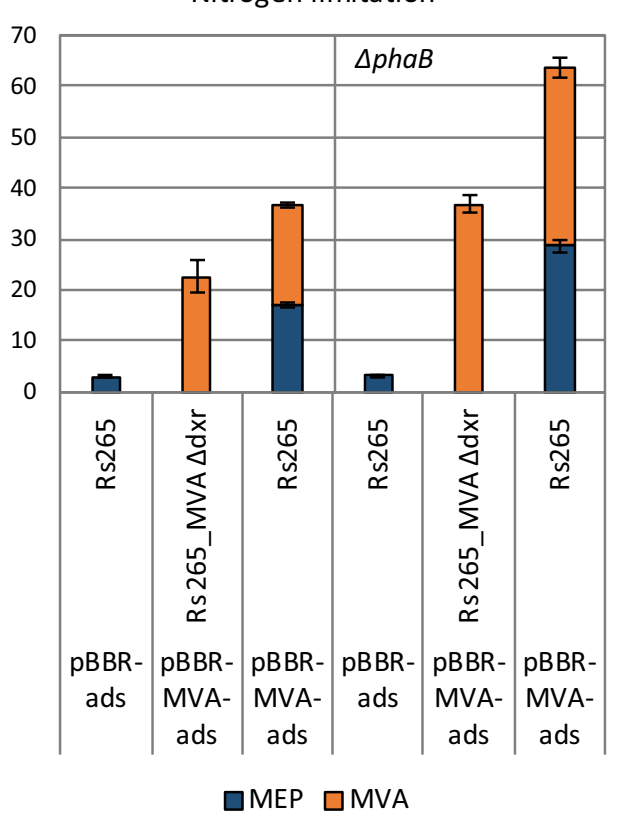

Fig. $3{ }^{13} \mathrm{C}$ metabolic flux ratio analysis of $R$. sphaeroides with different initial $\mathrm{C} / \mathrm{N}$ in the medium. The values are obtained from parallel labelling cultivation experiment. The isoprenoid flux ratios obtained were multiplied for the yield on biomass obtained for some of the strains of Fig. 2 . a Nitrogen excess conditions: enough nitrogen is provided to support cell division until depletion of glucose. $\mathbf{b}$ Nitrogen limitation: nitrogen will be depleted from the medium before glucose, and cell division is expected to stop and allow PHB accumulation 
plasmid (Fig. 3a, Table 4). Moreover, the capacity of the MVA pathway in the Rs265_LphaB and Rs265MVA_ $\Delta d x r \Delta p h a B$ strains was even further enhanced by the $p h a B$ deletion, as made obvious by comparison to strains that still contain the phaB gene (Fig. 3a, Table 4). In contrast, the flux through the native MEP pathway remained unaffected by the phaB deletion. Thus, under nitrogen excess conditions, deletion of the phaB gene results in an increase of the isoprenoid flux exclusively via the MVA pathway (Table 4).

We further studied the isoprenoid flux ratio under nitrogen-limited conditions (Fig. 3b). The Rs265:pBBR$a d s$ and Rs265_LphaB:pBBR-ads strains, which rely exclusively on the MEP pathway for isoprenoid production, did not show any increase in the amorphadiene/ biomass ratio when compared to nitrogen excess conditions (Fig. 3b). In contrast, the amorphadiene/biomass ratio for the strains that express only the MVA pathway was increased threefold when compared to this value under nitrogen excess conditions (Rs265-MVA_ $\Delta d x r$ and Rs265-MVA_ $\Delta d x r \Delta p h a B$ strains harbouring pBBRMVA-ads plasmid, Fig. 3). Interestingly, for the strains that express both pathways (Rs265:pBBR-MVA-ads and Rs265_AphaB:pBBR-MVA-ads strains, Fig. 3b), there was only a minor increase of the MVA pathway capacity in the Rs265_ $\triangle p h a B$ strain when compared to nitrogen excess conditions (Table 4). On the other hand, the MEP pathway capacity increased by $80 \%$ for the Rs 265 strain (from $9.4 \pm 0.3$ to $16.9 \pm 0.4 \mathrm{mg}$ amorphadiene $\cdot \mathrm{g}$ of biomass ${ }^{-1}$ ) and by $300 \%$ for the Rs265_ $\Delta$ phaB strain (from $9.2 \pm 1.3$ to $28.7 \pm 1.3 \mathrm{mg}$ amorphadiene $\cdot \mathrm{g}$ of biomass ${ }^{-1}$ ). Thus, for the dual-pathway strain, the increase of the amorphadiene/biomass ratio under nitrogen limitation conditions is attributed to the endogenous MEP pathway (Table 4).

Amorphadiene biosynthesis during resting cells conditions Under nitrogen-limited conditions a short exponential growth phase occurred, and therefore a short growthassociated amorphadiene production phase could not be avoided. This resulted in non-linear growth and production kinetics, making it difficult to assess yields (mg amorphadiene.g glucose $\mathrm{e}^{-1}$ ) and productivities (mg amorphadiene $\cdot \mathrm{L}^{-1} \cdot \mathrm{h}^{-1}$ ). In order to focus exclusively on growth-uncoupled production, and to obtain linear kinetics, we decided to assess amorphadiene production during resting cell conditions in nitrogen-free medium. This cultivation setup simulates the production phase of a two-stage fermentation setup where growth and production are separated.

Since deletion of phaB and expression of the MVA pathway increased production during nitrogen limitation, we reasoned to assess the amorphadiene production levels in the presence of also an active MEP pathway. Therefore, we further cultivated the strains Rs265, Rs265_ $\Delta p h a B$, Rs265-MVA_ $\Delta d x r$ and Rs265MVA_ $\Delta d x r \Delta p h a B$ under resting cells condition. All these strains contained the pBBR-MVA-ads plasmid (Fig. 4a).

A linear increase was observed in the $\mathrm{OD}_{600}$ of the strains with a functional PHB biosynthetic pathway (Rs265 and Rs265-MVA_ $\Delta d x r$, Fig. 4b). This trend is known to be associated with the accumulation of this storage compound [29], and it is associated with cell expansion rather than cell division. Accordingly, the corresponding $\triangle p h a B$ strains (Rs265_ $\Delta p h a B$ and Rs265MVA_ $\Delta d x r \Delta p h a B)$ did not show any increase in $\mathrm{OD}_{600}$. Glucose consumption (Fig. 4c, Additional file 1: Fig. S2), $\mathrm{pH}$ and amorphadiene concentrations (Fig. 4d, e) were followed over time. A decrease in the $\mathrm{pH}$ of the Rs265_ $\Delta p h a B$ and Rs265-MVA_ $\Delta d x r \Delta p h a B$ strains was observed (Fig. 4d), which can be explained by the secretion of organic acids upon prevention of PHB accumulation, mainly of pyruvate and 2-oxoglutarate (Fig. 4f).

Amorphadiene samples were collected over time from all the cultures (Fig. 4e), and yields and productivities were calculated for the first $24 \mathrm{~h}$ (Fig. 4g-i). The corresponding values for the Rs265 strain were the lowest among the tested strains. Deletion of phaB (Rs265_AphaB) resulted in a twofold increase of the amorphadiene/biomass ratio (Fig. 4g, Additional file 1: Table S2), the volumetric productivity (Fig. 4h) and the yield on glucose (Fig. 4i) compared to the Rs265 strain. Also, inactivation of the endogenous MEP pathway in the Rs265-MVA_ $\Delta d x r$ strain resulted in an increase of those values compared to Rs265 strain (Fig. 4h, i). Hence, inactivation of either the PHB production pathway or the endogenous MEP pathway stimulates

Table 4 Isoprenoid flux ratio calculated for the Rs265 and Rs265_AphaB strains harbouring the pBBR-MVA-ads plasmid after growth on different initial nitrogen concentrations

\begin{tabular}{|c|c|c|c|c|c|c|c|c|}
\hline \multirow[t]{3}{*}{ Genomic background } & \multicolumn{4}{|c|}{ Isoprenoid flux ratios } & \multicolumn{4}{|c|}{ Pathway capacities (mg g biomass ${ }^{-1}$ ) } \\
\hline & \multicolumn{2}{|c|}{ Nitrogen excess } & \multicolumn{2}{|c|}{ Nitrogen limitation } & \multicolumn{2}{|c|}{ Nitrogen excess } & \multicolumn{2}{|c|}{ Nitrogen limitation } \\
\hline & MEP & MVA & MEP & MVA & MEP & MVA & MEP & MVA \\
\hline Rs265 & $0.29 \pm 0.01$ & $0.71 \pm 0.01$ & $0.46 \pm 0.01$ & $0.54 \pm 0.1$ & $9.4 \pm 0.3$ & $23.0 \pm 0.3$ & $16.9 \pm 0.4$ & $19.9 \pm 0.5$ \\
\hline Rs265_4phaB & $0.24 \pm 0.03$ & $0.76 \pm 0.03$ & $0.45 \pm 0.02$ & $0.55 \pm 0.03$ & $9.2 \pm 1.3$ & $29.3 \pm 1.3$ & $28.7 \pm 1.3$ & $35.0 \pm 1.9$ \\
\hline
\end{tabular}




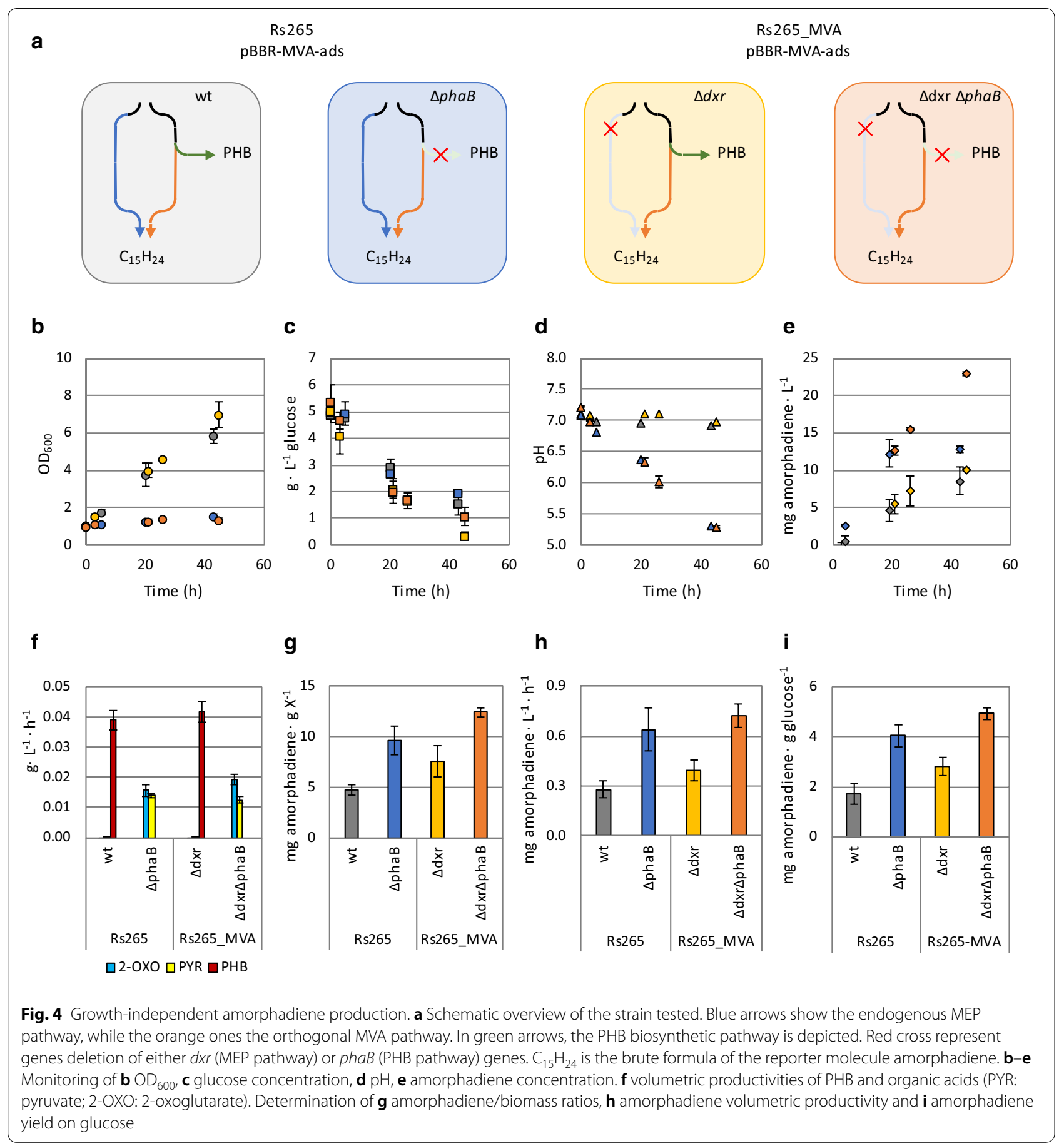

growth-independent production. Combined inactivation of the MEP and PHB production pathways (Rs265MVA_ $\Delta d x r \Delta p h a B$ strain) allowed this strain to reach the highest amorphadiene/biomass ratio, volumetric amorphadiene productivity (Fig. 4h) and yield on glucose (Fig. 4i). All these values were 2.5 -fold higher in the Rs265-MVA_ $\Delta d x r \Delta p h a B$ strain, compared to the Rs265 control strain. Hence, deletion of the endogenous MEP and PHB biosynthetic pathways resulted in the best metabolic setup for exploiting non-growing conditions for amorphadiene production. 


\section{Discussion}

Strain optimization for improved bioproduction often relies on strategies that couple production to microbial growth [19, 37]. Nevertheless, an emerging approach for metabolic engineering strategies is the one of dissociating production and growth [26]. Following this view, in this work we engineered the isoprenoid and the PHB biosynthetic pathways in $R$. sphaeroides. Therefore, we could demonstrate that isoprenoid biosynthesis, a typically growth-coupled type of metabolism in microorganisms, can be uncoupled from biomass production by means of rational metabolic engineering.

A previous work indicated that isoprenoid synthesis is strictly growth-coupled via the endogenous MEP pathway [29]. In the same study, the authors reported the first TRY parameters for this species, on a defined medium. They demonstrated that cultivation conditions can affect TRY values. By co-expressing both isoprenoid pathways, the yield of amorphadiene on glucose was highest under micro-aerobic conditions, reaching a value of about $0.005 \mathrm{~mol} \cdot \mathrm{mol}$ glucose $\mathrm{e}^{-1}$ [29]. This value is still considerably low compared to the maximum theoretical yield that could be obtained from glucose $(0.285 \mathrm{~mol} \cdot \mathrm{mol}$ glucose $\left.\mathrm{e}^{-1}\right)$. Moreover, the maximal amorphadiene productivity was attained during exponential growth and reached $2 \mathrm{mg} \cdot \mathrm{L}^{-1} \cdot \mathrm{h}^{-1}$. We applied nitrogen-limited conditions to a strain relying only on this isoprenoid pathway, but this did not result in increased amorphadiene/biomass ratio (Fig. 2b). We reasoned that targeting the storage compound (PHB) synthesis could increase the flux via the MEP pathway during this condition. We attempted to enhance the isoprenoid flux via this pathway because it presents the highest theoretical yield on glucose. Nevertheless, inactivation of the PHB synthetic pathway did not result in any improvement (Fig. 2b), thereby indicating that the endogenous MEP pathway is inhibited during non-growing conditions.

Differently from the MEP pathway, the MVA pathway is non-native in $R$. sphaeroides. Therefore, despite its lower theoretical yield, this heterologous pathway is expected to be less affected by endogenous regulation. Introduction of a heterologous MVA pathway was described to allow isoprenoid synthesis also during nongrowing conditions [29]. Here, we confirmed that nitrogen-limited conditions increased the amorphadiene/ biomass ratio in a strain relying exclusively on the MVA pathway (Fig. 2d). Moreover, inactivation of PHB synthesis by targeting phaB increased amorphadiene production when the MVA pathway was present (Fig. 2c, d). Because the current production of isoprenoids in $R$. sphaeroides is far from the maximum theoretical yield, the beneficial effect of MVA pathway expression on the overall isoprenoid flux compensated its lower theoretical yield. In order to calculate titers, rates and yields (TRY) of growth-independent amorphadiene synthesis, we performed cultivation under resting cells condition (Fig. 4). The experimental data confirmed that exclusive isoprenoid flux via the MVA pathway combined with inactivation of PHB synthesis results in maximal TRY values (Fig. 4e, g-i).

${ }^{13} \mathrm{C}$ metabolic flux ratio analysis was performed to understand the interaction between the two isoprenoid pathways during nitrogen limitation. The analysis indicated that in the dual-pathway strain the endogenous MEP pathway capacity is substantially enhanced during nitrogen limitation (Fig. 3b). Therefore, presence of the MVA pathway helps in deregulating the endogenous MEP pathway during nitrogen limitation.

Despite the increase in flux, only a small part of the carbon that originally went to $\mathrm{PHB}$ production could be redirected to amorphadiene. Organic acids-especially pyruvate and 2-oxoglutarate-were excreted instead. The accumulation of these two organic acids indicates that their downstream reactions, catalysed by the pyruvate dehydrogenase and 2-oxoglutarate dehydrogenase complexes, respectively, were inhibited by the inability to produce $\mathrm{PHB}$. These reactions require input of free CoA (Fig. 1), the co-enzyme released by the conversion of AACoA into PHB. We therefore speculate that knocking out either phaB or phaC1 and phaC2 decreased the availability of free CoA, that the capacity of the mevalonate pathway was insufficient to remedy this, and that this low CoA availability resulted in the accumulation of both pyruvate and 2-oxoglutarate. Possibly, secretion of $3 \mathrm{HB}$ after deletion of phaC1 and phaC2 allowed to release free CoA from the intermediate 3HB-CoA.

PHB synthesis results in [38]: (i) carbon storage, (ii) regeneration of $\mathrm{NADP}^{+}$and (iii) regeneration of free CoA for cellular homeostasis. Knocking out the ability to produce $\mathrm{PHB}$ should therefore increase the availability of precursors (AA-CoA) and NADPH for the MVA pathway. The relatively small increase in flux through the MVA pathway indicated that this pathway benefited from the increased amounts of AA-CoA and NADPH, but its capacity is limiting isoprenoid production. One of the rate-determining enzymes of the heterologous pathway is HMG-CoA reductase, already described as crucial enzyme for enhancing isoprenoid flux [15, 39]. We speculate that improving the catalytic efficiency of this enzyme might allow the MVA pathway to benefit from the higher availability of NADPH and AA-CoA. Such an improvement could allow to increase carbon flux towards isoprenoids, while reducing by-products secretion. Therefore, rational engineering strategies can build upon the findings of this work for further improving growthindependent isoprenoid biosynthesis in $R$. sphaeroides. 


\section{Conclusions}

In this work, we assessed the contribution of the MEP and MVA pathways to amorphadiene biosynthesis under different culturing conditions. We confirmed that application of the heterologous MVA pathway holds potential for growth-independent production. In a dual-pathway strain, enhancement of the endogenous MEP pathway capacity was confirmed during nitrogenlimited conditions via ${ }^{13} \mathrm{C}$ metabolic flux ratio analysis.

Nevertheless, isoprenoid synthesis during resting cells condition was limited by the presence of an active endogenous MEP pathway. On the other hand, exclusive isoprenoid flux via the MVA pathway increased amorphadiene synthesis during this condition. Additionally, prevention of PHB synthesis via phaB resulted in the highest TRY values for growth-independent amorphadiene production.

Ultimately, this work proposed a metabolic engineering design for increasing growth-independent isoprenoid biosynthesis in $R$. sphaeroides, while providing novel insights about the interaction occurring between the two isoprenoid pathways.

\section{Supplementary information}

Supplementary information accompanies this paper at https://doi. org/10.1186/s13068-020-01765-1.

Additional file 1. Additional figures and tables.

\begin{abstract}
Abbreviations
PHB: Poly- $\beta$-hydroxybutyrate; MEP: 2-Methyl-D-erythritol 4-phosphat; MVA: Mevalonate; CRISPR-Cas: Clustered regularly inter-spaced short palindromic repeats-CRISPR-associated proteins; PYR: Pyruvate; CoA: Coenzyme-A; NADPH: Dihydronicotinamide-adenine dinucleotide phosphate; Ac-CoA: Acetyl-COA; AA-CoA: Acetoacetyl-CoA; HMG-CoA: Hydroxymethylglutaryl-CoA; 3-HB-CoA: 3-Hydroxybutyryl-COA; 3HB: 3-Hydroxybutyrate; 2-OXO: 2-Oxoglutarate; phaB: Acetoacetylreductase (gene); $d x r$ : 1-Deoxy-D-xylulose 5-phosphate reductoisomerase (gene); ads: Amorphadiene synthase (gene).
\end{abstract}

\section{Acknowledgements}

The authors thank Isobionics BV for providing the Rhodobacter sphaeroides Rs 265 strain used in this study. Any request for the strain and its derivatives should be directed to Isobionics BV. Moreover, we thank Rosyida Arifah for technical assistance in generating the Rs265-MVA_AdxrAphaB strain, and Frank E. Fluitman for providing the Rs265_4phaC1 4 phaC2 strain.

\section{Authors' contributions}

EO, IM, SWMK and RAW designed the work. EO, IM, WP JB and MD conducted, analysed, and interpreted the experiments. EO and IM drafted and wrote the manuscript. All authors read and approved the final manuscript.

\section{Funding}

This project was financially supported by The Netherlands Ministry of Economic Affairs, via a public-private NWO-Green Foundation for sustainable production and supply chains in agriculture and horticulture $(870.15 .130,2015 / 05279 / A L W)$. This project has received funding from the European Research Council (ERC) under the European Union's Horizon 2020 research and innovation programme, grant agreement No. 834279.

\section{Availability of data and materials}

The dataset supporting the conclusions of this article are included within the article and in the additional file.

\section{Ethics approval and consent to participate}

Not applicable.

\section{Consent for publication}

Not applicable.

\section{Competing interests}

The authors declare that they have no competing interests.

\section{Author details}

${ }^{1}$ Bioprocess Engineering, Wageningen University, Droevendaalsesteeg 1, 6708 PB Wageningen, The Netherlands. ${ }^{2}$ Laboratory of Microbiology, Wageningen University, Stippeneng 4, 6708 WE Wageningen, The Netherlands. ${ }^{3}$ Wageningen Plant Research, 6700AA Wageningen, The Netherlands. ${ }^{4}$ Physical Chemistry and Soft Matter, Wageningen University, Stippeneng 4, 6708 WE Wageningen, The Netherlands. ${ }^{5}$ Wageningen Food \& Biobased Research, 6708WG Wageningen, The Netherlands. ${ }^{6}$ Present Address: Systems and Synthetic Metabolism Group, Max Planck Institute of Molecular Plant Physiology, Am Mühlenberg 1, 14476 Potsdam, Germany. ${ }^{7}$ Present Address: Helmholtz Institute for RNA-based Infection Research (HIRI), Helmholtz-Centre for Infection Research (HZl), 97080 Würzburg, Germany.

Received: 17 February 2020 Accepted: 7 July 2020

Published online: 13 July 2020

\section{References}

1. Ajikumar PK, Tyo K, Carlsen S, Mucha O, Phon TH, Stephanopoulos G. Terpenoids: opportunities for biosynthesis of natural product drugs using engineered microorganisms. Mol Pharm. 2008;5(2):167-90.

2. Schempp FM, Drummond L, Buchhaupt M, Schrader J. Microbial cell factories for the production of terpenoid flavor and fragrance compounds. J Agric Food Chem. 2018;66(10):2247-58.

3. Park SY, Yang D, Ha SH, Lee SY. Metabolic engineering of microorganisms for the production of natural compounds. Adv Biosyst. 2018;2:1-41.

4. Kirby J, Keasling JD. Biosynthesis of plant isoprenoids: perspectives for microbial engineering. Annu Rev Plant Biol. 2009;60(1):335-55.

5. Mewalal R, Rai DK, Kainer D, Chen F, Kulheim C, Peter GF, et al. Plantderived terpenes: a feedstock for specialty biofuels. Trends Biotechnol. 2016;35(3):227-40.

6. Niu FX, Lu Q, Bu YF, Liu JZ. Metabolic engineering for the microbial production of isoprenoids: carotenoids and isoprenoid-based biofuels. Synth Syst Biotechnol. 2017;2(3):167-75.

7. Kuzuyama T, Seto H. Two distinct pathways for essential metabolic precursors for isoprenoid biosynthesis. Proc Jpn Acad Ser B. 2012;88(3):41-52.

8. Lombard J, Moreira D. Origins and early evolution of the mevalonate pathway of isoprenoid biosynthesis in the three domains of life. Mol Biol Evol. 2011;28(1):87-99.

9. Vranová E, Coman D, Gruissem W. Network analysis of the MVA and MEP pathways for isoprenoid synthesis. Annu Rev Plant Biol. 2013;64(1):665-700.

10. Martin VJJ, Pitera DJ, Withers ST, Newman JD, Keasling JD. Engineering a mevalonate pathway in Escherichia coli for production of terpenoids. Nat Biotechnol. 2003;21(7):796-802.

11. Yuan $L Z$, Rouvière PE, LaRossa RA, Suh W. Chromosomal promoter replacement of the isoprenoid pathway for enhancing carotenoid production in Escherichia coli. Metab Eng. 2006;8(1):79-90.

12. Ajikumar PK, Xiao WH, Tyo KEJ, Wang Y, Simeon F, Leonard E, et al. Isoprenoid pathway optimization for Taxol precursor overproduction in Escherichia coli. Science. 2010;330(6000):70-4.

13. Li Q, Fan F, Gao X, Yang C, Bi C, Tang J, et al. Balanced activation of IspG and IspH to eliminate MEP intermediate accumulation and improve isoprenoids production in Escherichia coli. Metab Eng. 2016;2017(44):13-21.

14. Anthony JR, Anthony LC, Nowroozi F, Kwon G, Newman JD, Keasling JD. Optimization of the mevalonate-based isoprenoid biosynthetic pathway 
in Escherichia coli for production of the anti-malarial drug precursor amorpha-4, 11-diene. Metab Eng. 2009;11(1):13-9.

15. Pitera DJ, Paddon CJ, Newman JD, Keasling JD. Balancing a heterologous mevalonate pathway for improved isoprenoid production in Escherichia coli. Metab Eng. 2007;9(2):193-207.

16. Orsi E, Beekwlider J, van Gelder D, van Houwelingen A, Eggink G, Kengen SWM, et al. Functional replacement of isoprenoid biosynthetic pathways in Rhodobacter sphaeroides. Microb Biotechnol. 2020;13(4):1082-93.

17. Kannenberg EL, Poralla K. Hopanoid biosynthesis and function in bacteria. Naturwissenschaften. 1999;86(4):168-76.

18. Volkman JK. Sterols in microorganisms. Appl Microbiol Biotechnol. 2003;60(5):495-506.

19. Von Kamp A, Klamt S. Growth-coupled overproduction is feasible for almost all metabolites in five major production organisms. Nat Commun. 2017:8:1-10.

20. Reyes $\mathrm{LH}$, Gomez JM, Kao KC. Improving carotenoids production in yeast via adaptive laboratory evolution. Metab Eng. 2014;21:26-33.

21. Klamt S, Mahadevan R, Hädicke O. When do two-stage processes outperform one-stage processes? Biotechnol J. 2018;13(2):1700539.

22. Willrodt C, Hoschek A, Bühler B, Schmid A, Julsing MK. Decoupling production from growth by magnesium sulfate limitation boosts de novo limonene production. Biotechnol Bioeng. 2016;113(6):1305-14.

23. Tsuruta H, Paddon CJ, Eng D, Lenihan JR, Horning T, Anthony LC, et al. High-level production of amorpha-4,11-diene, a precursor of the antimalarial agent artemisinin, in Escherichia coli. PLOS ONE. 2009;4(2):e4489.

24. Li S, Jendresen CB, Nielsen AT. Increasing production yield of tyrosine and mevalonate through inhibition of biomass formation. Process Biochem. 2016;51(12):1992-2000.

25. Zhang L, Liu L, Wang KF, Xu L, Zhou L, Wang W, et al. Phosphate limitation increases coenzyme Q10 production in industrial Rhodobacter sphaeroides HY01. Synth Syst Biotechnol. 2019;4(4):212-9.

26. Pandit AV, Srinivasan S, Mahadevan R. Redesigning metabolism based on orthogonality principles. Nat Commun. 2017:8:1-11.

27. Su A, Chi S, Li Y, Tan S, Qiang S, Chen Z, et al. Metabolic redesign of Rhodobacter sphaeroides for lycopene production. J Agric Food Chem. 2018;66:5879-85.

28. Beekwilder J, van Houwelingen A, Cankar K, van Dijk ADJ, de Jong RM, Stoopen G, et al. Valencene synthase from the heartwood of Nootka cypress (Callitropsis nootkatensis) for biotechnological production of valencene. Plant Biotechnol J. 2014;12(2):174-82.
29. Orsi E, Folch PL, Monje-López VT, Fernhout BM, Turcato A, Kengen SWM, et al. Characterization of heterotrophic growth and sesquiterpene production by Rhodobacter sphaeroides on a defined medium. J Ind Microbiol Biotechnol. 2019;46(8):1179-90.

30. Orsi E, Beekwilder J, Peek S, Eggink G, Kengen SWM, Weusthuis RA. Metabolic flux ratio analysis by parallel $13 \mathrm{C}$ labeling of isoprenoid biosynthesis in Rhodobacter sphaeroides. Metab Eng. 2020;57:228-38.

31. Mougiakos I, Orsi E, Ghiffari MR, De Maria A, Post W, Adiego-Perez B, et al. Efficient Cas9-based genome editing of Rhodobacter sphaeroides for metabolic engineering. Microb Cell Fact. 2019;18(204):1-13.

32. Kim M, Baek J, Lee JK. Comparison of $\mathrm{H} 2$ accumulation by Rhodobacter sphaeroides KD131 and its uptake hydrogenase and PHB synthase deficient mutant. Int J Hydrogen Energy. 2006;31:121-7.

33. Ryu M-H, Hull NC, Gomelsky M. Metabolic engineering of Rhodobacter sphaeroides for improved hydrogen production. Int J Hydrogen Energy. 2014;39(12):6384-90.

34. Shimizu T, Teramoto H, Inui M. Introduction of glyoxylate bypass increases hydrogen gas yield from acetate and L-glutamate in Rhodobacter sphaeroides. Appl Environ Microbiol. 2019;85(2):1-17.

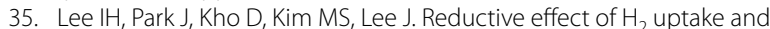
poly- $\beta$-hydroxybutyrate formation on nitrogenase-mediated $\mathrm{H} 2$ accumulation of Rhodobacter sphaeroides according to light intensity. Appl Microbiol Biotechnol. 2002;60(1-2):147-53.

36. Yang C, Gao X, Jiang Y, Sun B, Gao F, Yang S. Synergy between methylerythritol phosphate pathway and mevalonate pathway for isoprene production in Escherichia coli. Metab Eng. 2016;37:79-91.

37. Mans R, Daran JMG, Pronk JT. Under pressure: evolutionary engineering of yeast strains for improved performance in fuels and chemicals production. Curr Opin Biotechnol. 2018:50:47-56.

38. Leaf TA, Srienc F. Metabolic modeling of polyhydroxybutyrate biosynthesis. Biotechnol Bioeng. 1998;57(5):557-70.

39. Sasaki Y, Eng T, Herbert RA, Trinh J, Chen Y, Rodriguez A, et al. Engineering Corynebacterium glutamicum to produce the biogasoline isopentenol from plant biomass hydrolysates. Biotechnol Biofuels. 2019;12(1):41.

\section{Publisher's Note}

Springer Nature remains neutral with regard to jurisdictional claims in published maps and institutional affiliations.
Ready to submit your research? Choose BMC and benefit from:

- fast, convenient online submission

- thorough peer review by experienced researchers in your field

- rapid publication on acceptance

- support for research data, including large and complex data types

- gold Open Access which fosters wider collaboration and increased citations

- maximum visibility for your research: over $100 \mathrm{M}$ website views per year

At BMC, research is always in progress.

Learn more biomedcentral.com/submissions 